[14] T. R. Larson, L. I. Moskowitz, and J. W. Wright, "A note on SAR imagery of the ocean," IEEE Trans. Antennas Propagat., vol, AP-24, pp. 393-394, May 1976.

[15] R. K. Raney, "Synthetic aperture imaging radar and moving targets," IEEE Trans. Aerosp. Electron. Syst., vol. AES-7, pp. 499-505, 1971.

[16] F. Belanger, "Effect of random phase errors on synthetic arrays," Arizona Electron. Eng. Memo., No. 238, 1966.

[17] F. Belanger, "Parabolic phase errors and defocussing", Goodyear Aerosp. AEEM-373 (Arizona Elec. Eng. Memo.), 1969.

[18] C. T. Swift and L. R. Wilson, "Synthetic aperture radar imaging of ocean waves," submitted to IEEE Trans. Antennas Propagat.

[19] R. O. Harget, Synthetic Aperture Radar Systems. New York: Academic, 1970.

[20] R. A. Shuchman and J. S. Zelenka, "Processing of ocean wave data from a synthetic aperture radar," Boundary Lay'er Meteorol., vol. 13, pp. 181-192, 1978.

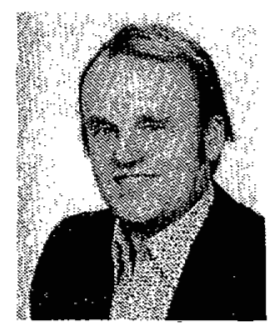

Werner R. Alpers was born in Hamburg, Germany. He studied physics at the Universities' of Hamburg, Zurich, and Wisconsin. He received the M.S. degree in physics from the University of Wisconsin in 1964 and the Ph.D. degree in theoretical physics from the University of Hamburg, Hamburg, Germany, in 1967.

From 1968 to 1970 he worked in space physics at the European Space Research Institute at Frascati, Italy, and from 1970 to 1973 at the Max-Planck-Institute for Physics and Astrophysics in Munich,
Germany. Since 1973 he has been with the University of Hamburg, and the Max-Planck-Institute for Meteorology in Hamburg, Germany, working on radio oceanographic research problems. His current research activities are focused on measuring ocean surface waves and currents using active microwave sensors.

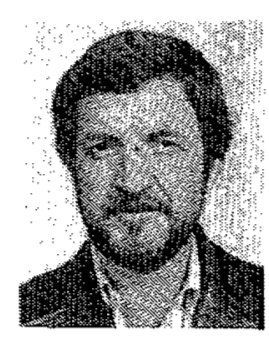

Clifford L. Rufenach (S'62-M'70) was born in Ronan, MT, in 1936. He received the B.S. and M.S. degrees from Montana State University, Bozeman, in 1962 and 1963 and the Ph.D. degree from the University of Colorado, Boulder, in 1971 , all in electrical engineering.

From 1963 to 1966 he worked on radio propagation problems at the Stanford Research Institule and from 1967 until present he has been working for the Environmental Research Laboratories of the National Oceanographic and Atmospheric Administration (NOAA), Boulder, CO. His work at NOAA includes research on the radio physics of the ionosphere and interplanetary medium and, more recently, on remote sensing of the ocean surface using satellite and aircraft sensors.

Dr. Rufenach is a member of Tau Beta $\mathrm{P} i$, Sigma Xi, the American Geophysical Union, and URSI.

\title{
Synthesis of Antenna Arrays with Spatial and Excitation Constraints
}

\author{
N. BALAKRISHNAN, P. K. MURTHY, AND S. RAMAKRISHNA
}

\begin{abstract}
Synthesis of antenna arrays subject to spatial and excitation constraints to yield arbitrarily prescribed patterns in both the mean-squared and minimax sense are discussed. The spatial constraints may require that the interelement spacings be greater than a prescribed value or that the element locations lie within a specified region. The excitation constraints are of the form where the current-taper ratio is constrained to be less than or equal to a prescribed value. The technique employed consists of reducing the constrained optimization problem into an unconstrained one by the use of simple transformations of the independent variables. In such cases where explicit transformations are not available, a created response surface technique (CRST) has been used to convert the constrained optimization problem into a series of unconstrained optimizations. The optimization has been carried out using a nonlinear simplex algorithm. Numerical examples are given wherein both the linear and circular arrays are synthesized subject to constraints.
\end{abstract}

Manuscript received July 6, 1978; revised February 27, 1979.

The authors are with the Department of Aeronautical Engineering, Indian Institute of Science, Bangalore 560012 , India.

\section{INTRODUCTION}

$\mathbf{T}$ THE PROBLEM of synthesizing nonuniformly spaced antenna arrays has been studied quite extensively, and comprehensive accounts of the techniques employed have appeared in books [1], [2]. These techniques include the Fourier series expansion, methods of approximation theory, and interpolation.

Very few attempts have been made where synthesis has been carried out subject to constraints on element positions, currents, or pattern characteristics such as sidelobe level or beamwidth. Schuman and Strait [3] have described an iterative approach to synthesize arrays whose elements are constrained to lie within specified limits. Sandrin, Glatt, and Hague [4] have reported a method employing a computerized multivariate search technique wherein constraints on sidelobe levels, beamwidths, and interelement spacings may be imposed. Perini [5] has employed the steepest descent technique to design arrays with large interelement spacings. Schjaer-Jacobson and Madsen [6] have described a nonlinear minimax 
algorithm based on the successive linear approximation technique and have discussed the possibility of constraints on spacings. Harrington and Mautz [7] have employed the Lagrange multiplier technique to synthesize a given radiation pattern with a constraint on the source norm.

This paper presents a general method of synthesizing arbitrarily prescribed patterns subject to spatial and excitation constraints. The spatial constraints may, for example, require that the interelement spacings be greater than a prescribed value or that the element positions be within specified limits. The excitation constraint may require that the current-taper ratio be less than or equal to a prescribed value. The technique employed consists of reducing the constrained optimization problem into an unconstrained one by the use of suitable transformations of the independent variables [8]. In cases where such transformations are not possible, a created response surface is defined [8], [9] to convert the constrained optimization problem into a series of unconstrained optimization problems. The mean-squared and minimax error criteria have been employed. A number of examples have been worked out to illustrate the effectiveness of this method in systematically synthesizing arrays with various constraints.

\section{FORMULATION}

\section{A. Geometry of the Arrays}

The array factor of an $N$-element array of isotropic radiators is given by [1]

$$
f_{s}[\vec{I}, \bar{\alpha}, \bar{r} ; \theta, \varphi]=\sum_{k=1}^{N} I_{k} e^{j \alpha_{k}} e^{j \beta\left(\bar{r} \cdot \bar{r}_{k}\right)},
$$

where $I_{k}, \alpha_{k}$, and $\bar{r}_{k}$ are the current, phase, and position vectors of the $k$ th element, $\bar{r}$ is the position vector of the point of observation, and $\beta$ is the propagation constant. For a centersymmetric linear unequally spaced array with $N=2 n$ elements the array factor becomes

$$
f_{s}[\bar{I}, \bar{\alpha}, \bar{X} ; \varphi]=\sum_{k=1}^{n} 2 I_{k} \cos \left(\beta x_{k} \cos \varphi+\alpha_{k}\right)
$$

For a circular array of $N$ isotropic elements equally spaced around a circle of radius $a$, the array factor in the plane of this array is given by [10]

$$
E(\varphi)=2 \sum_{k=-n}^{n} I_{k} \cos \left[\beta a \cos \left(\varphi+\frac{2 k \pi}{N}\right)+\alpha_{k}\right],
$$

where $N=4 n, \alpha_{k}=\alpha_{-k}=-\alpha_{k^{\prime}}=-\alpha_{-k^{\prime}}$ for $k=0,1,2, \cdots$, $n-1$, and $\alpha_{n}=\alpha_{-n}=0$.

Let $f_{d}$ be the desired pattern. Adopting the $L_{p}$ norm [11] over a finite point set of population $m$, equally distributed over the domain of fit, the error between the synthesized and desired patterns is given by

$$
\sigma_{p}=\left\|f_{s}-f_{d}\right\|_{p}=\left[\sum_{i=1}^{m}\left|f_{s, i}-f_{d, i}\right|^{p} w_{i}\right]^{1 / p}
$$

where $\left[w_{i}\right]$ is a set of positive weights. $w_{i}$ are chosen depending on the relative emphasis to be placed on the errors at different parts of the domain of fit. In the absence of any such priorities, $w_{i}$ may be taken to be constant. The synthesis problem now consists of determining the various array parameters $\bar{I}, \bar{X}$, and $\bar{\alpha}$ so that $\sigma_{p}$ is minimum subject to the spatial and excitation constraints.

\section{B. Constraints}

a) It is often necessary to impose a constraint on the interelement spacings to minimize the mutual coupling effects or because the extent of the individual element aperture is larger than the nominal interelement spacing. For an array with an even number of elements the constaint may then be expressed in the following form:

$$
x_{1} \geqslant D / 2, \quad\left(x_{i}-x_{i-1}\right) \geqslant D, \quad i=2,3, \cdots, n,
$$

where $x_{k}$ and $D$ are in wavelengths.

The resulting constrained optimization problem may now be converted into one without constraints by the use of the following transformation:

$$
x_{1}=\frac{D}{2}+\left(x_{1}{ }^{\prime}\right)^{2}, \quad x_{2}=\left(\frac{D}{2}+D\right)+\left(x_{1}^{\prime}\right)^{2}+\left(x_{2}^{\prime}\right)^{2} .
$$

And, in general

$$
x_{i}=\left(i-\frac{1}{2}\right) D+\sum_{k=1}^{i}\left(x_{k}{ }^{\prime}\right)^{2}, \quad i=1,2, \cdots, n
$$

For an array with an odd number of elements (6) becomes

$$
x_{i}=(i-1) D+\sum_{k=1}^{i-1}\left(x_{k}{ }^{\prime}\right)^{2}, \quad i=2,3, \cdots,(N-1) / 2 .
$$

Substituting (6) in (4) allows minimization to be carried out with the new primed variables, and it is readily seen that the constraints are always satisfied.

Another type of constraint on spacings usually imposed is the one requiring the elements to lie within a specified interval. This could be stated mathematically in the following form:

$$
a_{i} \leqslant x_{i} \leqslant b_{i}, \quad i=1,2, \cdots, n
$$

The transformation to be used in this case is

$$
x_{i}=a_{i}+\left(b_{i}-a_{i}\right) \sin ^{2} x_{i}^{\prime}
$$

b) It is sometimes necessary to constrain the current taper to be within specified limits. That is,

$$
I_{i} \leqslant I \pm C, \quad i=1,2, \cdots, n .
$$

It is easily verified that the transformation of the form

$$
I_{i}=I+C \sin I_{i}^{\prime}
$$

will transform the constrained space into an unconstrained one.

c) In general, there may be situations where the transformation method described earlier may be unsuitable. This may 
TABLE I

SYNTHESIS OF GAUSSIAN PATTERN $N=6, \alpha_{k}=0, I_{k}=1 / N$ FOR ALL $k$

\begin{tabular}{|c|c|c|c|c|c|c|c|}
\hline \multirow[b]{2}{*}{ SI. No. } & \multirow[b]{2}{*}{ Norm } & \multirow[b]{2}{*}{ Constraints } & \multicolumn{3}{|c|}{ Element Positions in $\lambda$} & \multirow{2}{*}{$\begin{array}{l}\text { Sidelobe Level } \\
\text { in } \mathrm{dB}\end{array}$} & \multirow{2}{*}{$\begin{array}{c}\text { Mean-Squared } \\
\text { Error }\end{array}$} \\
\hline & & & $x_{1}$ & $x_{2}$ & $x_{3}$ & & \\
\hline 1 & $L_{\infty}$ & $\begin{array}{l}\text { No constraints; } \\
\qquad D=0\end{array}$ & 0.252 & 0.503 & 1.121 & -20.19 & - \\
\hline 2 & $L_{2}$ & $\begin{array}{c}\text { No constraints; } \\
D=0\end{array}$ & 0.26 & 0.480 & 1.085 & -20.39 & 0.0042 \\
\hline 3 & $L_{2}$ & $D=0.50$ & 0.25 & 0.750 & 1.29 & -13.3 & 0.0151 \\
\hline 4 & $L_{2}^{2}$ & $D=0.75$ & 0.3997 & 1.165 & 1.916 & -11.88 & 0.0491 \\
\hline 5 & $L_{2}^{2}$ & $D=1.0$ & 0.507 & 2.291 & 4.113 & -2.6 & 0.1235 \\
\hline 6 & $\begin{array}{l}L_{2}^{2} \\
L_{4}\end{array}$ & $D=1.0$ & 0.517 & 1.531 & 3.175 & -4.8 & - \\
\hline
\end{tabular}

arise due to the absence of either a simple transformation or an explicit relationship to describe the constraint. Such a situation can arise, for example, when it is required that the sidelobe level in a given region does not exceed a specified value. Such constraints may either be equalities or inequalities and may be described mathematically as

$$
\begin{array}{ll}
\phi_{k}(\bar{I}, \bar{\alpha}, \bar{X} ; \varphi) \leqslant 0, & k=1,2, \cdots, s \\
\psi_{k}(\bar{I}, \bar{\alpha}, \bar{X} ; \varphi)=0, & k=1,2, \cdots, q .
\end{array}
$$

In such cases a penalty, namely, the created response surface technique (CRST), is employed. In this method a new objective function can be defined as [9]

$$
\sigma_{p}{ }^{\prime}=\sigma_{p}+r \sum_{k=1}^{s} \phi_{k}+\sum_{k=1}^{q} \frac{\left(\psi_{k}\right)^{2}}{\sqrt{r}}
$$

where $r$ is a positive constant whose initial value is normally taken as unity. It is seen from (12) that penalties are levied whenever the constraints are violated. The procedure now consists of carrying out a minimization of (12). The value of $r$ is increased by a constant factor, and with the solution of the preceding iteration as the starting point (12) is minimized again. This iteration is carried out until no further reduction is obtained. Thus, this method converts the original constrained optimization problem into a series of unconstrained optimizations, with each iteration descending down a created response surface. A particular use of this method is in the systhesis of arrays using the $L_{\infty}$ norm; a faster convergence is effected when the equiripple property of the best minimax approximation is introduced as a constraint.

The error criteria and the constraints are thus merged to form an unconstrained objective function. The transformed objective function is in general nonlinear and its derivatives difficult to compute. Hence, use of a sequential search technique, namely, the simplex method of Nelder and Mead [12], is resorted to. A flowchart from which the simplex method can easily be programmed in the Fortran IV language is given in [12]. The simplex method sets up $n+1$ points called simplex in an $n$-dimensional space. It gropes towards the minimum by flipping or contracting the simplex. The logic used is based on an evaluation of the function at each corner of the simplex.

\section{NUMERICAL ILLUSTRATIONS}

Several examples have been worked out, and the results are presented in this section. In order to keep the computations simple only symmetric arrays with a real array factor are considered for synthesis. The number of elements for the linear arrays has been chosen to be six and for the circular array 36. Although the examples considered below are for arrays with an even number of elements, the method is equally applicable to arrays with an odd number of elements. A computer program in the Fortran IV language has been written to implement the optimization technique described earlier.

\section{A. Equally Excited Arrays}

1) Broadside Arrays: The Gaussian pattern has been chosen for synthesis of broadside arrays and is given by

$$
\begin{aligned}
f_{d}\left(\varphi_{i}\right) & =\exp \left[-15\left(\varphi_{i}-\frac{\pi}{2}\right)^{2}\right] \\
\varphi_{i} & =(i-1) \frac{\pi}{2} / 180, \quad i=1,2, \cdots, 181 .
\end{aligned}
$$

Since the pattern is symmetrical about $\varphi=\pi / 2$, it is sufficient to synthesize the pattern in the range $(0, \pi / 2)$.

The Gaussian pattern is synthesized by the equally excited center-symmetric linear broadside (i.e., $\alpha_{k}=0$ for all $k$ ) array. For the unconstrained case both $L_{2}$ and $L_{\infty}$ norms are used as the error criteria. Constraints on element positions as defined in (6) with $D=0.5,0.75$, and 1.0 are imposed and the array synthesized using the $L_{2}$ norm as the error measure. The results are given in Table $I$. The radiation patterns are shown in Fig. 1. The results obtained here for both the unconstrained cases by this method are identical to the results of Kumar and Murthy [13] who employed the perturbation technique.

It is clear from the table that as the severity of the constraint is increased (increasing value of $D$ ) the sidelobe level increases. The case with $D=1.0$ is noteworthy, as this method offers a systematic way of designing arrays with interelement spacings larger than a wavelength, a problem tackled earlier by Unz [14]. In this case the grating lobe is only $-2.6 \mathrm{~dB}$. It is known that by choosing the $L_{p}$ norm as the error criterion with larger values of $p$, relatively greater weights are placed on the peaks of the error curve. This fact is used to reduce the grating-lobe level of the six-element array by choosing a value of 4 for $p$. The grating-lobe level fell in this case to $-4.8 \mathrm{~dB}$. Further increase in $p$ will continue to cause the grating lobe to fall further. However, this will result in increased sidelobe levels elsewhere. In the limiting case $(p \rightarrow \infty)$ the sidelobe structure will tend to be equiripple as is to be expected from Polya's algorithm [11].

In order to assess how effectively the grating lobe could be controlled the same pattern is again synthesized by a 12element array. The grating lobe in this case has further come down to $-6.3 \mathrm{~dB}$. The element positions (in wavelengths) for 


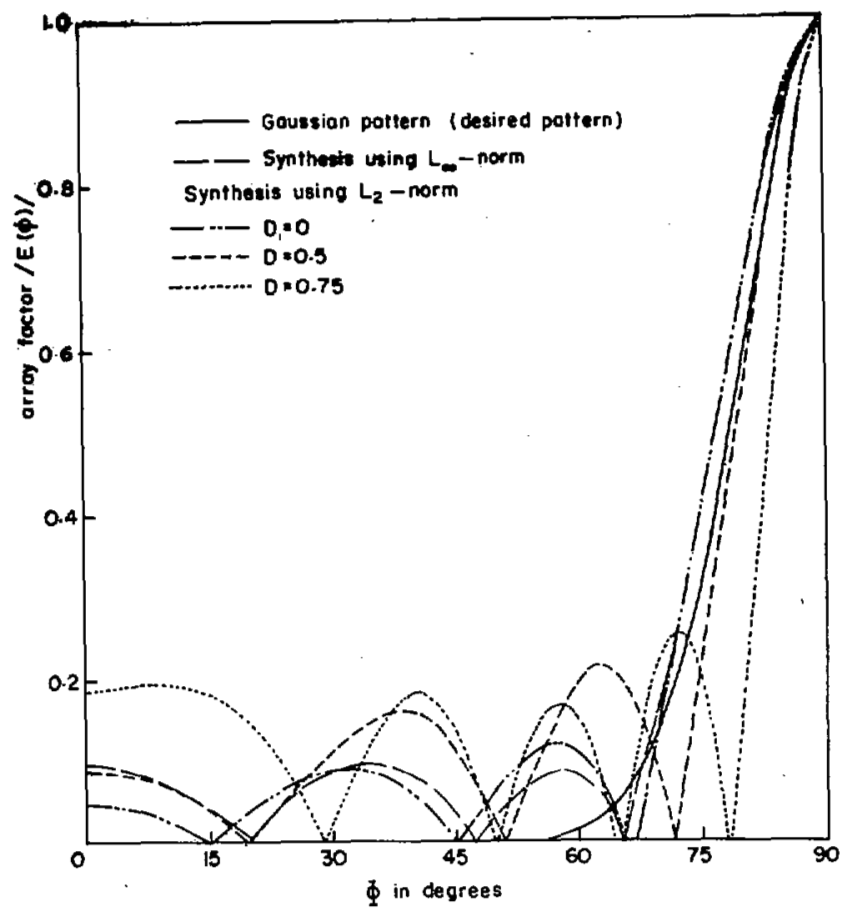

(a)

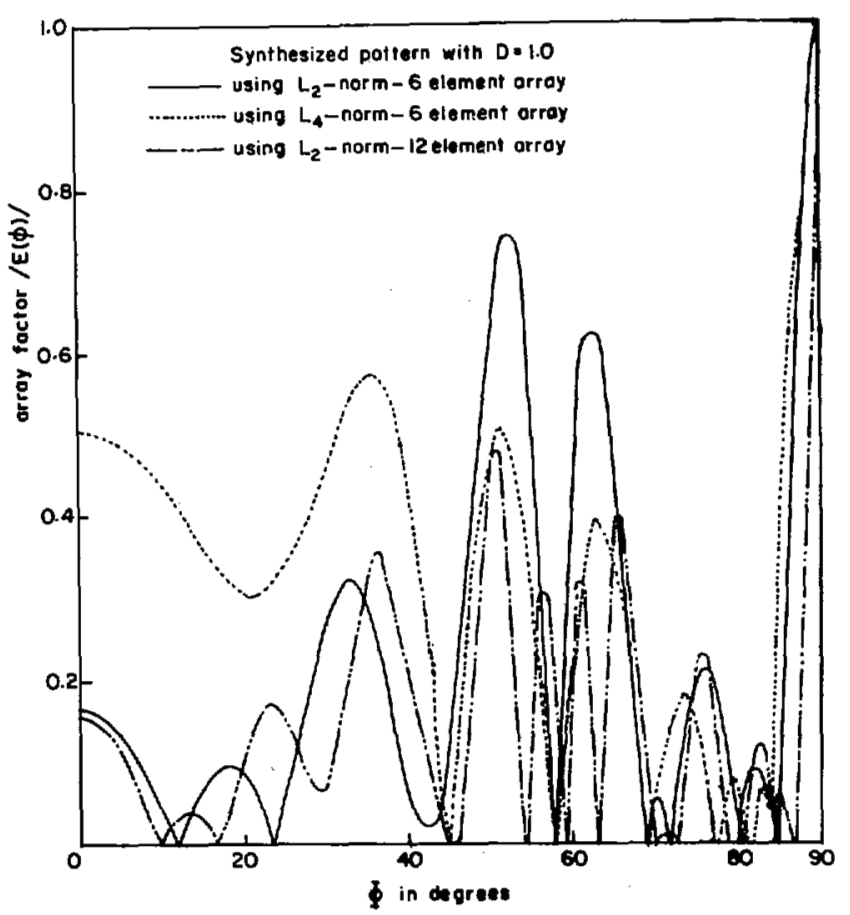

(b)

Fig. 1. Synthesis of equally excited broadside arrays.

this case are given as

$$
\begin{aligned}
& x_{1}=0.51, \quad x_{2}=1.5, \quad x_{3}=3.28, \quad x_{4}=4.29, \\
& x_{5}=6.26, \quad x_{6}=8.03
\end{aligned}
$$

2) Endfire Array: For the case of endfire arrays the desired pattern has been chosen to coincide with the main lobe of a $0.2-\lambda$ spaced six-elemerit Hansen-Woodyard endfire array and zero everywhere else. For such a situation we have

$$
\begin{aligned}
f_{d}\left(\varphi_{i}\right) & =0, \quad \text { for } \frac{-\pi}{N-1}-2 \beta d \leqslant \psi_{i} \leqslant-\frac{\pi}{2 N} \\
& =\frac{1}{N} \frac{\sin \left(N \psi_{i} / 2\right)}{\sin \psi_{i} / 2}, \quad \text { for }-\frac{\pi}{2 N} \leqslant \psi_{i} \leqslant \frac{\pi}{N-1},
\end{aligned}
$$

where

$$
\begin{aligned}
& \psi_{i}=\beta d \cos \varphi+\alpha_{\mathrm{HW}}, \\
& \alpha_{\mathrm{HW}}=-\beta d-\frac{\pi}{N-1}, \quad \varphi_{i}=(i-1) \frac{\pi}{180}
\end{aligned}
$$

The above pattern has been synthesized by a center-symmetric six-element array. The $L_{2}$ norm has been employed as the error criterion. The synthesis has first been carried out by varying the element positions only. In this case the phases are taken to be uniformly progressive. That is,

$$
\alpha_{k}=-\beta x_{k}, \quad k=1,2,3 .
$$

The minimum interelement spacing $D$ is taken as 0.25 . The final element positions in wavelengths have been found to be

$$
x_{1}=0.218, \quad x_{2}=0.650, \quad x_{3}=1.038
$$

The sidelobe level and directivity have been found to be -11.3 $\mathrm{dB}$ and 9.5, respectively. The corresponding figures for the $0.2-\lambda$ spaced Hanson-Woodyard array are $-6.62 \mathrm{~dB}$ and 8.29. It can be seen that there is an improvement of $4.68 \mathrm{~dB}$ in the sidelobe level with an increase in the directivity as well. Also, the mainbeam efficiency, which is a measure of super directivity [2], is 100 percent for the unequally spaced case as compared to 26.3 percent for the Hansen-Woodyard case.

The Hansen-Woodyard pattern has also been synthesized by varying the phases alone but keeping the interelement spacing of $0.2 \lambda$. The sidelobe level and directivity for this case have been found to be $-15.4 \mathrm{~dB}$ and 10.08 , respectively. The synthesized patterns of the unequally spaced and nonuniformly phased arrays are shown in Fig. 2. The simplex algorithm in the unequally phased case converged to a set of phases of antenna currents $\left(\alpha_{1}=-0.9, \alpha_{2}=-2.19\right.$, and $\left.\alpha_{3}=1.94\right)$ which proved to be different from those obtained by using the perturbation technique [13] $\left(\alpha_{1}=-0.89, \alpha_{2}=-2.58\right.$, and $\alpha_{3}=-4.89$ ). This suggests the existence of multiple minima. A comparison of the mean-squared error values for the two methods (simplex: 0.0137 ; perturbation: 0.0987) establishes that the simplex method has yielded a better minimum and that the minimum to which the perturbation method has converged is a local one.

This example serves to illustrate the possibility of the existence of multiple minima. Owing to the complexity of the functions involved, the number of local minima will increase with the dimension $n$ of the problem. Thus, the probability of ending up at a local minimum will increase with $n$ irrespective of the method employed. However, the simplex method is intrinsically more resistant to convergence to local minima than most other methods by virtue of its having multiple $(n+1)$ starting points, with the result that the probability of one of them being close to the global minimum is higher. This 


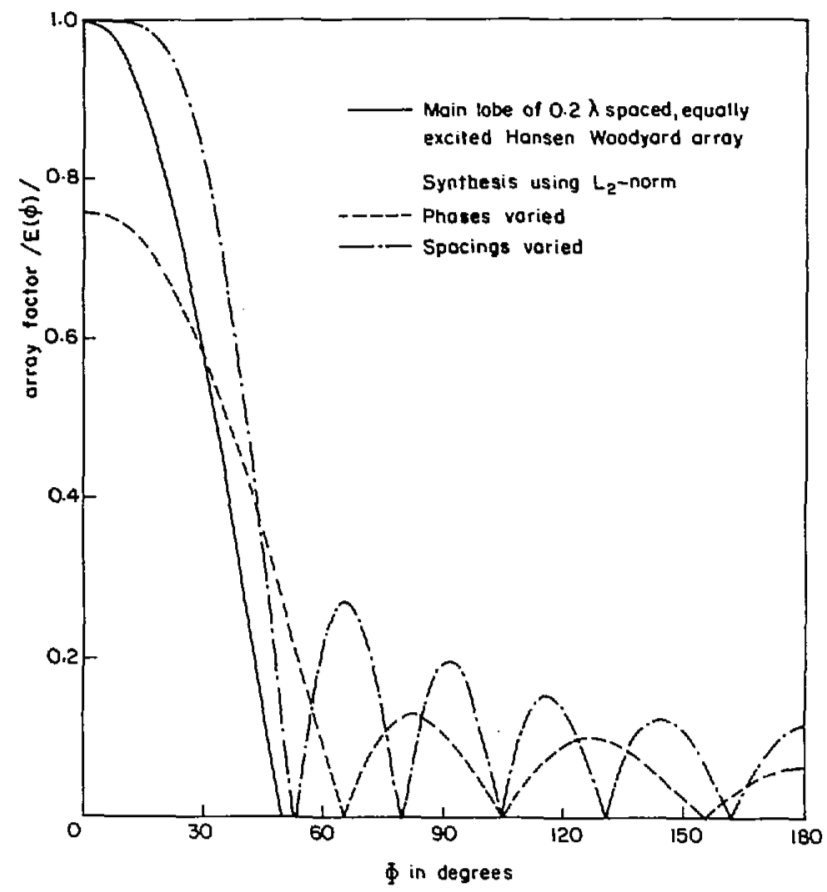

Fig. 2. Synthesis of equally excited endfire arrays.

probability can be further improved by initiating the search several times randomly over the domains of search. The interaction between increasing $n$ and an increased number of starting simplices is quite intricate, depending heavily on the type of function handled. It is difficult to relate quantitatively the probability of falling into a local minimum to the dimension $n$.

3) Circular Array: The desired pattern has been chosen to coincide with the main beam of the array factor of a 36element symmetric uniformly phased and equally excited array. From (3) this could be stated as

$$
f_{d}(\varphi)=\left\{\begin{array}{cc}
2 \sum_{k=-n}^{n} I_{k} \cos \left[\beta a \cos \left(\varphi+\frac{2 k \pi}{N}\right)+\alpha\right], \\
\text { for }-\varphi_{0} \leqslant \varphi \leqslant \varphi_{0} \\
0, \quad \text { everywhere else }
\end{array}\right.
$$

with $N=4 n=36, \beta a=9$, and where $\varphi_{0}$ is the first null of the array factor.

The circular array pattern has been synthesized employing $L_{\infty}$ norm as the error criterion by varying the phases of elements only. The final phases in radians corresponding to this case are

$$
\begin{aligned}
& \alpha_{0}=-9.0556, \quad \alpha_{1}=-7.7944, \quad \alpha_{2}=-8.0101, \\
& \alpha_{3}=-8.8440, \quad \alpha_{4}=-6.5510, \quad \alpha_{5}=-6.6894 \text {, } \\
& \alpha_{6}=-5.2576, \quad \alpha_{7}=3.8000, \quad \alpha_{8}=-1.6298 \text {, } \\
& \alpha_{9}=0 \text {. }
\end{aligned}
$$

The desired and synthesized patterns are shown in Fig. 3 . It can be seen that the sidelobe level has decreased to -12.12 $\mathrm{dB}$ from approximately $-8 \mathrm{~dB}$ for the uniform array.

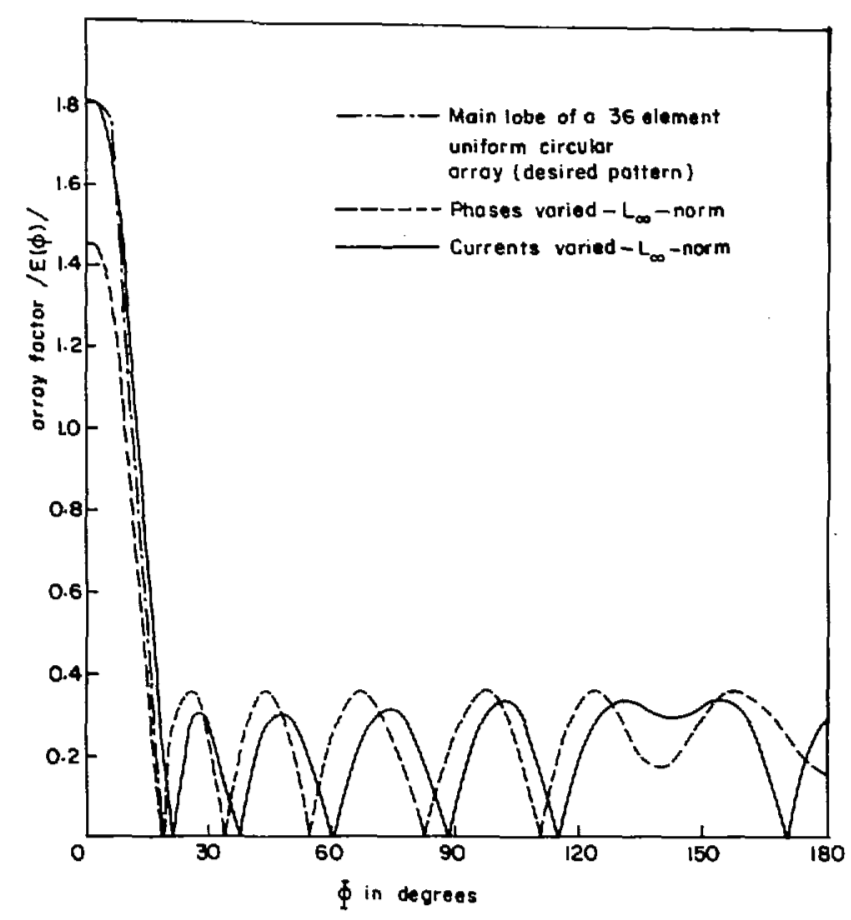

Fig. 3. Synthesis of circular arrays.

\section{B. Unequally Excited Arrays}

1) Broadside Array: The Gaussian pattern defined by (13) is synthesized with the $L_{2}$ norm as the error criterion by a sixelement center-symmetric linear array with both antenna currents and element positions allowed to vary. However, the currents are constrained to be within specified limits only, and the minimum interelement spacing $D$ is required to be at least 0.75 . That is,

$$
-C+0.3333 \leqslant I_{k} \leqslant 0.3333+C, \quad D=0.75
$$

The values of $C$ have been chosen to be $0.2,0.1$, and 0.05 . The transformations (6) and (10) have been used and the minimization of the resulting objective function is carried out. The results are given in Table II. The Gaussian pattern has also been synthesized with no constraints on either the element currents or positions but employing both the $L_{2}$ and $L_{\infty}$ norms. These results are also included in Table II for the sake of comparison. The radiation patterns are given in Fig. 4 .

It may be seen from Table II that for decreasing values of $C$, the constraint on the current, the minimum mean-squared error between the desired and the synthesized patterns increases, resulting in higher sidelobe levels. The antenna currents and positions obtained for the unconstrained case employing the $L_{2}$ norm can be seen to concur with those obtained in [15]. The perturbation technique in [15] breaks down in the case of the $L_{\infty}$ norm and modifications are necessary, whereas with this method the minimum has been reached without any difficulty. Recently Streit [16] proved that the radiation patterns whose sidelobes are all of equal level are optimum in the sense that the Chebyshev arrays are. In other words, the beam width is the least obtainable for a given sidelobe level or viceversa. Thus, the radiation pattern corresponding to synthesis with the $L_{\infty}$ norm is the optimum pattern in this sense. 
TABLE II

SYNTHESIS OF GAUSSIAN PATTERN WITH $I$ AND $X$ VARIED $N=6, \alpha_{k}=0$ FOR ALL $k$

\begin{tabular}{|c|c|c|c|c|c|c|c|c|c|c|c|}
\hline \multirow{3}{*}{$\begin{array}{l}\text { Sl. } \\
\text { No. }\end{array}$} & \multirow[b]{3}{*}{ Norm } & \multicolumn{2}{|c|}{ Contraints } & \multirow{2}{*}{\multicolumn{3}{|c|}{ Element Currents }} & \multicolumn{3}{|c|}{ Element Positions } & \multirow{3}{*}{$\begin{array}{c}\left(\sigma_{2}\right)^{2} \times \\
10^{-2}\end{array}$} & \multirow{3}{*}{$\begin{array}{c}\text { Sidelobe } \\
\text { Level } \\
\text { (in db) }\end{array}$} \\
\hline & & Current & Spacings & & & & & (in $\lambda$ ) & & & \\
\hline & & C & $D$ & $I_{1}$ & $I_{2}$ & $I_{3}$ & $x_{1}$ & $x_{2}$ & $x_{3}$ & & \\
\hline 1 & $L_{\infty}$ & \multicolumn{2}{|c|}{ No constraints } & 0.5777 & 0.3221 & 0.0905 & 0.3447 & 1041 & 1.7577 & - & -41.3 \\
\hline 2 & $L_{2}$ & \multicolumn{2}{|c|}{ No constraints } & 0.577 & 0.324 & 0.089 & 0.349 & 1.036 & 1.746 & 0.0042 & -41.74 \\
\hline 3 & $L_{2}^{2}$ & 0.20 & 0.75 & 0.5330 & 0.3289 & 0.1339 & 0.377 & 1.1435 & 1.894 & 0.3746 & -22.66 \\
\hline 4 & $L_{2}^{2}$ & 0.10 & 0.75 & 0.4333 & 0.3338 & 0.2337 & 0.4 & 1.16 & 1.91 & 1.8232 & -18.35 \\
\hline 5 & $L_{2}^{2}$ & 0.05 & 0.75 & & 0.3011 & 0.2835 & 0.3827 & 1.1572 & 1.9074 & 3.0331 & -15.13 \\
\hline
\end{tabular}

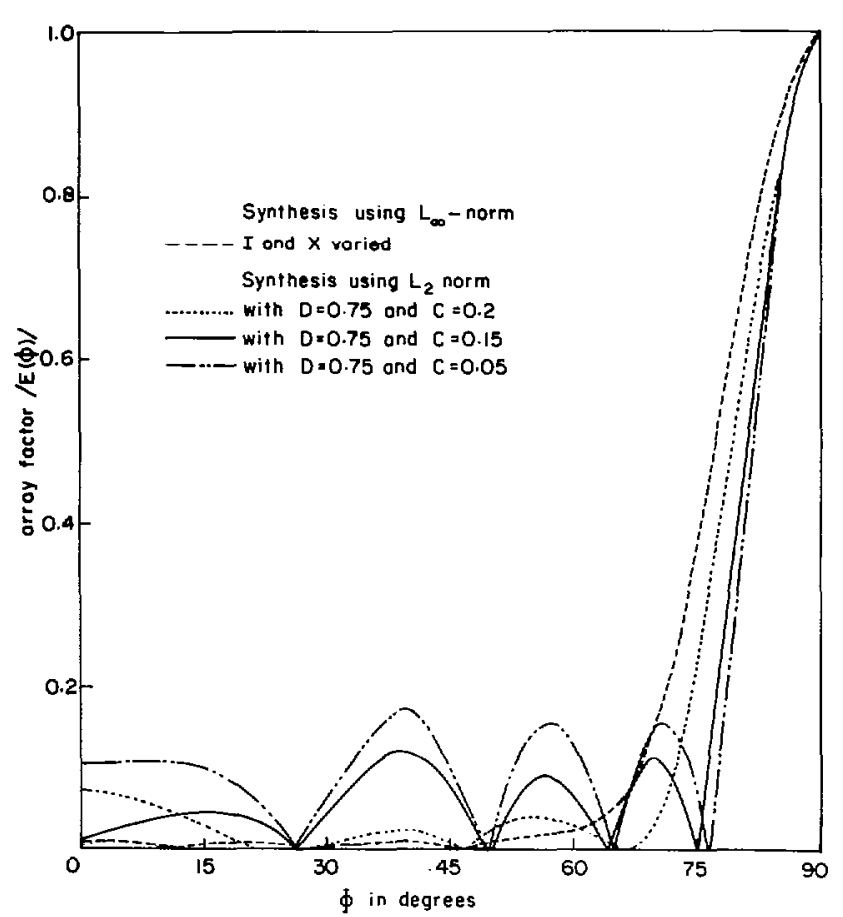

Fig. 4. Synthesis of unequally excited broadside arrays.

2) Circular Array: The circular array pattern defined by (16) has been synthesised employing the $L_{\infty}$ norm by varying the amplitudes of the element currents only. The currents corresponding to the amplitude-varied case are

$$
\begin{array}{lll}
I_{0}=0.2068, & I_{1}=0.1814, & I_{2}=0.1576, \\
I_{3}=0.0473, & I_{4}=0.0384, & I_{5}=0.0864, \\
I_{6}=0.0386, & I_{7}=0.0999, & I_{8}=0.0804, \\
I_{9}=0.0646 . &
\end{array}
$$

The synthesized pattern is shown in Fig. 3, and it may be noted that the sidelobe level is only $-14.52 \mathrm{~dB}$, though the main beam is somewhat broadened.

\section{CONCLUSION}

The value of the method described in this paper lies principally in the unusually wide class of constraints that can be handled by using the transform technique. However, to be meaningful it must be able to handle a significant number of variables. Examples with ten variables have been given. Calculations have been made with as many as 20 variables without any serious difficulties being encountered. The number has been limited by the computational facility (IBM 360/44) available to the authors. With better facilities the number that can be handled is likely to be much higher.

For simplex minimization problems the computational time is expected to vary approximately as $(n+1)^{2.11}[12]$ where $n$ is the number of variables handled. Our computations have corroborated this. There would, of course, be a constant multiplier depending on the complexity of the function and the speed of the computer.

\section{ACKNOWLEDGMENT}

The authors wish to thank Dr. P. R. Mahapatra for his valuable assistance in programming the simplex algorithm.

\section{REFERENCES}

[1] R. E. Collin and F. J. Zucker, Antenna Theory. New York: McGraw-Hill, 1969, part I, ch. 5-7.

[2] M. T. Ma, Theory and Applications of Antenna Arrays. New York: Wiley, 1974.

[3] H. K. Schuman and B. J. Strait, "On the design on unequally spaced arrays with nearly equal sidelobes," IEEE Trans. Antennas Propagat., vol. AP-16, p. 493, July 1968.

[4] W. A. Sandrin, C. R. Glatt, and D. S. Hauge, "Design of arrays with unequal spacing and partially uniform amplitude taper," IEEE Trans. Antennas Propagat., vol. AP-17, pp. 642-644, Sept. 1969.

[5] J. Perini, "Sidelobe reduction by beam shifting," IEEE Trans. Antennas Propagat., vol. AP-12, pp. 791-792, Nov. 1964.

[6] H. Schjaer-Jacobson and K. Madsen, "Snythesis of non-uniformly spaced arrays using a general nonlinear mini-max optimization method," IEEE Trans. Antennas Propagat., vol. AP-24, pp. 501-506, July 1976.

[7] J. R. Mautz and R. F. Harrington, "Computational methods for antenna pattern synthesis," IEEE Trans. Antennas Propagat., vol. AP-23, pp. 507-512, July 1975.

[8] M. J. Box, D. Davis, and W. H. Swann, Nonlinear Optimization Techniques. Edinburgh: Oliver and Boyd, 1969.

[9] A. V. Fiacco and G. P. McCormic, Nonlinear Programming: Sequential Unconstrained Minimization Techniques. New York: Wiley, 1968.

[10] N. Goto and Y. Tsundo, "Sidelobe reduction of circular arrays with a constant excitation amplitude," IEEE Trans. Antennas Propagat., vol. AP-25, pp. 896-898, Nov. 1977.

[11] J. R. Rice, The Approximation of Functions-Vol. 1 and Vol. 2. Reading, MA; Addison-Wesley, 1964.

[12] J. A. Nelder and R. Mead, "A simplex method for function minimization," Comp. J., vol. 7, pp. 308-314, 1965.

[13] A. Kumar and P. K. Murthy, "Synthesis of equally excited arrays," IEEE Trans. Antennas Propagat., vol. AP-25, pp. 425428, May 1977.

[14] H. Unz, "Nonuniform arrays with spacings larger than one wavelength," IRE Trans. Antennas Propagat., vol. AP-10, no. 5, pp. 647-648, Sept. 1962.

[15] P. K. Murthy and A. Kumar, "Synthesis of linear antenna ar- 
rays," IEEE Trans. Antennas Propagat., vol. AP-24, pp. 865870, Nov, 1976.

[16] R. Streit, "Sufficient conditions for the existence of optimum beam patterns for unequally spaced linear arrays with an example," IEEE Trans. Antennas Propagat., vol. AP-23, pp. 112115, Jan. 1975.

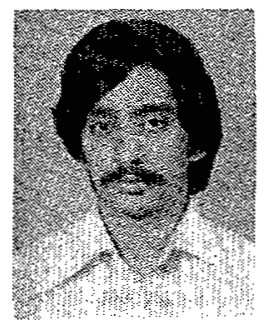

N. Balakrishnan completed the Bachelor of Engineering in electronics and communication from the University of Madras, Madras, India, in 1972.

In 1973 he joined the Department of Aeronautical Engineering, Indian Institute of Science, Bangalore, India, where he is currently employed as a Scientific Officer. His fields of interests are digital electronics, solid-state microwaves, microcomputers, and antenna array theory.
P. K. Murthy, biography and photograph not available at time of publication.

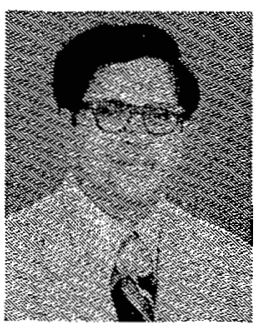

S. Ramakrishna was born in Visakhapatnam, Andhra Pradesh, India, on September 17, 1938. He graduated from Osmania University in 1957 and received the M.S. degree in nuclear physics and the Ph.D. degree in radio astronomy from the Indian Institute of Science, Bangalore, India.

From 1962 to 1968 he was a Senior Scientific Officer with the Defense Electronics Research Laboratory, Hyderabad, India, where he was Head of the Space Electronic Division. In 1969 he worked for L.R.D.E., Bangalore, in the Radar Division. He joined the Department of Aeronautical Engineering at the Indian Institute of Science in 1969 where he is currently a Professor. His research interests are missile guidance and instrumentation, radar systems, avionics, atmospheric physics, and antenna theory.

\section{Communications}

\section{Some Notes on ELF Earth-Ionosphere Waveguide Daytime Propagation Parameters}

PETER R. BANNISTER, MEMBER, IEEE

\begin{abstract}
The transverse-electromagnetic (TEM) propagation constants for extremely low frequency (ELF) daytime propagation in the earth-ionosphere waveguide have been calculated for frequencies of 5-2000 $\mathrm{Hz}$. The recently developed theory of Greifinger and Greifinger and the Wait very low frequency (VLF) exponential ionospheric-conductivity profile have been used in the analysis. It is shown that the resulting values of ELF attenuation rate, phase velocity, and ionosphericreflection height are in excellent agreement with the measured data.
\end{abstract}

\section{INTRODUCTION}

Recently, Greifinger and Greifinger [1] have derived a simple-form approximate expression for the transverse-electromagnetic (TEM) eigenvalues (propagation constants) for extremely low frequency (ELF) propagation in the earth-ionosphere waveguide under conditions where anisotropy due to the earth's magnetic field may be neglected. Strictly speaking, the method is applicable only to daytime ionospheres or sufficiently disturbed nighttime ionospheres. In principle, however, it could be extended to handle anisotropy as well. The authors demonstrated that eigenvalues obtained by their method were in excellent agreement with numerically calculated eigenvalues

Manuscript received September 27, 1978; revised May 9, 1979.

The author is with the New London Laboratory, Naval Underwater Systems Center, New London, CT 06320.
(Field [2]) for "single scale-height" or "two scale-height" ionospheric-conductivity profiles. (For details on the usual methods of calculating eigenvalues for ELF propagation in the earthionosphere waveguide see Galejs [3] or Wait [4].)

The Greifingers' method of determining approximate eigenvalues depends on the details on the ionospheric-conductivity profile only in two limited altitude ranges. The lower of these regions is the neighborhood of the altitude $h_{0}$, at which $\sigma=\omega \epsilon_{0}$ (i.e., where the displacement and conduction currents become equal). The upper region is the neighborhood of the altitude $h_{1}$, at which $4 \omega \mu_{0} \sigma \zeta_{1}^{2}=1$, where $\zeta_{1}$ is the conductivity scale height at the altitude $h_{1}$. (This is the altitude at which $\omega \tau_{0}=1$, where $\tau_{0}$ is the magnetic-diffusion time through a conductivity scale height.)

It is the purpose of this communication to apply the Greifingers' theory to the famous Wait exponential ionosphericconductivity profile [4], [5], a profile that has been widely used in determining very low frequency (VLF) propagation parameters. It will be shown that the resulting values of ELF attenuation rate $\alpha$, phase velocity $v$, and effective ionosphericreflection height $h_{\text {eff }}$ are in excellent agreement with the measured data.

\section{THEORY}

The Greifingers' expression for the eigenvalue $S_{0}$ is (for $\exp (+i \omega t)$ time dependence)

$$
S_{0}^{2}=1-C_{0}^{2} \sim \frac{\left(h_{1}-i \pi \zeta_{1} / 2\right)}{\left(h_{0}+i \pi \zeta_{0} / 2\right)}
$$

where $S_{0}$ and $\mathcal{C}_{0}$ are the sine and cosine; respectively, of the complex waveguide incidence angle; $h_{0}$ is the altitude where $\sigma=\omega \epsilon_{0} ; h_{1}$ is the altitude where $4 \omega \mu_{0} \sigma \zeta_{1}^{2}=1$; and $\zeta_{0}$ and 